\section{SOI: $1.1 /$ TAS $\quad$ DOI: $10.15863 /$ TAS International Scientific Journal Theoretical \& Applied Science}

p-ISSN: 2308-4944 (print) ｅ-ISSN: 2409-0085 (online)

Year: 2015 Issue: 03 Volume: 23

Published: $30.03 .2015 \quad$ http://T-Science.org

SECTION 4. Computer science, computer engineering and automation.

\section{Victoriya Victorovna Konkina}

Postgraduate

Tambov State Technical University

Tambov, Russia

konkina-tstu@yandex.ru

Denis Sergeevich Solovjev

Candidate of Engineering Sciences, Assistant

Tambov State Technical University

Tambov, Russia

solovjevdenis@mail.ru

\title{
MATHEMATICAL MODELLING OF ELECTROPLATING IN REVERSE MODE FOR THE MULTIANODE BATH
}

Abstract: A new method of reducing the nonuniformity of electrolytic plating, which consists in the act of combining the reversing current mode with turning off of the coincident anodic sections in multi anode bath has been proposed. The formulation of the problem of optimal control of the described process is given. The effectiveness of the proposed method is demonstrated in comparison with the classical mode of reversing in monolithic anode bath based on the example of electroplating in 9 anodic nickel plating bath on the flat shaped product.

Key words: multianode bath, reverse mode, mathematic modeling, nonuniformity of electrolytic plating.

Language: Russian

Citation: Konkina VV, Solovjev DS (2015) MATHEMATICAL MODELLING OF ELECTROPLATING IN REVERSE MODE FOR THE MULTIANODE BATH. ISJ Theoretical \& Applied Science 03 (23): 59-62.

Soi: http://s-o-i.org/1.1/TAS*03(23)12 Doi: crostef http://dx.doi.org/10.15863/TAS.2015.03.23.12

\section{МАТЕМАТИЧЕСКОЕ МОДЕЛИРОВАНИЕ НАНЕСЕНИЯ ГАЛЬВАНИЧЕСКОГО ПОКРЫТИЯ В РЕВЕРСИВНОМ РЕЖИМЕ ДЛЯ МНОГОАНОДНОЙ ВАННЫ}

Аннотация: Предложен новый способ снижения неравномерности покрытия, заключающийся 6 сочетании режима реверсирования тока с отключением соответствующих анодных секций в многоанодной ванне. Приводится постановка задачи оптимального управления описываемым прочессом. Продемонстрирована эффективность предлагаемого способа по сравнению с классическим режимом реверсирования в ванне с монолитным анодом на примере нанесения гальванического покрытия в 9-ти анодной гальванической ванне никелирования на изделие плоской формы.

Ключевые слова: многоанодная ванна, реверсивный режим, математическое моделирование, неравномерность покрытия.

Снижение

неравномерности гальванического покрытия - одна из важных задач автоматизации гальванических процессов. Для решения данной задачи в промышленности используют различные способы, одним из которых является применение ванн со многими анодами. К перспективному способу получения равномерного распределения гальванического покрытия можно отнести реализацию реверсивного режима в многоанодной ванне [1]. Однако, эффективность применения такого режима невозможна без разработки алгоритмов моделирования и оптимального управления предлагаемым процессом.

В рассматриваемом процессе классический монолитный анод разбивается на систему секций одинакового размера размерностью $M \times \mathrm{x} N$, размещенных в одной плоскости на равном расстоянии друг от друга, стенок ванны и напротив изделия-катода. Данная система секций подключается к программируемому источнику питания (к такому как «Пульсар Про», «Flex Kraft») через устройство, которое позволяет отключать требуемые анодные секции для «прямого» и «обратного» режимов 
реверсирования тока. Это приведет к снижению неравномерности распределения гальванического покрытия, так как за время протекания «прямого» и «обратного» тока скорость растворения металла покрытия на наиболее близких к анодным секциям поверхностях катода будет больше, чем на удаленных [2].

Математическая постановка задачи оптимального управления рассматриваемым процессом звучит следующим образом: найти матрицы активных анодных секций для «прямого» $I_{M x N}^{\text {прям* }}(\tau)$ и «обратного» $I_{M x N}^{\text {обр * }}(\tau)$ режимов реверсивного гальванического процесса, a также длительности $\tau^{\text {прям* }}, \tau^{\text {обр* }}$ таких периодов, доставляющие минимум критерию неравномерности распределения толщины наносимого покрытия по поверхности катода $S_{k}$ :

$$
\begin{aligned}
& R(\tau)^{*}=\frac{1}{\left\|S_{k}\right\|} \int_{S_{k}} \frac{\delta(x, y, z, \tau)-\delta^{\min }(\tau)}{\delta^{\min }(\tau)} \cdot d S_{k}+ \\
& +\frac{\delta^{\max }(\tau)-\delta^{\min }(\tau)}{\delta^{\min }(\tau)} \rightarrow \min
\end{aligned}
$$

при этом необходимо выполнение ограничений:

$$
\begin{gathered}
\delta^{\min }(T) \geq \delta^{z a d}, \\
T=v\left(\tau^{\text {прям }}+\tau^{\text {обр }}\right) \leq T^{\text {max }}, \\
\text { где } \quad \delta^{z a d}-\quad \text { заданная }
\end{gathered}
$$

гальванического покрытия; $\delta^{\text {min }}$ - минимальная толщина гальванического покрытия; $\delta^{\max }-$ максимальная толщина гальванического покрытия; $\quad T \quad$ - продолжительность гальванического процесса; $T^{\max }$ - максимальная продолжительность гальванического процесса; $x$, $y, z$ - координаты точки в пространстве гальванической ванны; v - количество периодов включения «прямого» и «обратного» тока.

Матрицы активных анодных секций для «прямого» и «обратного» режимов включения соответственно имеет вид:

$$
\begin{aligned}
I_{M x N}^{\text {прям }}(\tau) & =\left(\begin{array}{lll}
I_{1,1}^{\text {прям }}(\tau) \ldots & I_{1, n}^{\text {прям }}(\tau) \ldots & I_{1, N}^{\text {прям }}(\tau) \\
I_{m, 1}^{\text {прям }}(\tau) \ldots & I_{m, n}^{\text {прям }}(\tau) \ldots & I_{m, N}^{\text {прям }}(\tau) \\
I_{M, 1}^{\text {прям }}(\tau) \ldots & I_{M, n}^{\text {прям }}(\tau) \ldots & I_{M, N}^{\text {прям }}(\tau)
\end{array}\right)(4) \\
I_{M x N}^{\text {обр }}(\tau) & =\left(\begin{array}{lll}
I_{1,1}^{\text {обр }}(\tau) \ldots & I_{1, n}^{\text {обр }}(\tau) \ldots & I_{1, N}^{\text {обр }}(\tau) \\
I_{m, 1}^{\text {oбр }}(\tau) \ldots & I_{m, n}^{\text {обр }}(\tau) \ldots & I_{m, N}^{\text {обр }}(\tau) \\
I_{M, 1}^{\text {обр }}(\tau) \ldots & I_{M, n}^{\text {обр }}(\tau) \ldots & I_{M, N}^{\text {обр }}(\tau)
\end{array}\right) \quad(5)
\end{aligned}
$$

В работе [3] для рассматриваемого процесса предложена математическая модель содержащая закон Фарадея, Ома в дифференциальной форме, а так же дифференциальное уравнение Лапласа в частных производных, которое описывает распределение потенциала в пространстве многоанодной гальванической ванны. Конфигурация гальванической ванны задается геометрической моделью рецепторного типа.

Особенностью

разработанной математической модели является то, что в граничные условия для дифференциального уравнения Лапласа в частных производных добавлена проверка включения соответствующей анодной секции для «прямого» и «обратного» режима реверсирования.

Задача поиска оптимального управления предложенным процессом представляет собой частично целочисленную задачу нелинейного бинарного программирования, что обусловлено возможностью принятия только бинарных значений для элементов матриц [4]. Исходя из известных недостатков существующих подходов к решению задач целочисленного программирования (методы ветвей и границ, комбинаторные), авторами выбран метод полного перебора для поиска оптимальной конфигурации системы активных анодных секций для «прямого» и «обратного» режима, при этом отыскание длительностей включения режима осуществляется методом Хука-Дживса [5, 6]. Выбор этого метода обусловлен требованиями к минимальному количеству вычислений критерия неравномерности покрытия посредством решения уравнения математической модели для поиска данных управляющих воздействий.

Приведем пример математического моделирования и оптимального управления для гальванического процесса нанесения никелевого покрытия для плоского стального катода $S_{k}$ : квадратной формы, размером $L_{1} \times L_{2}=30 \times 30 \mathrm{~cm}$ (площадью $900 \quad \mathrm{~cm}^{2}$ ) и 9 -ти анодной гальванической ванны, функционирующей в режиме реверсирования тока. Гальваническая ванна размерами $X \mathrm{x} Y \mathrm{x} Z=40 \times 40 \times 45$ см заполнена по уровню 40 см стандартным электролитом Уоттса, имеющего следующий состав, г/л: $\mathrm{NiSO}_{4} \cdot 7 \mathrm{H}_{2} \mathrm{O}-280 \ldots 300, \mathrm{NiCl}_{2} \cdot 6 \mathrm{H}_{2} \mathrm{O}-50 \ldots 70$, $\mathrm{H}_{3} \mathrm{BO}_{3}-30 \ldots 40[7,8]$. В качестве анодных секций $S_{a_{m, n}}$ используются квадратные никелевые

пластины размерами 10х10 см, с одинаковыми межанодными расстояниями в 2 см. При этом расстояние между системой анодных секций и катодом составляет $30 \mathrm{~cm}$.

Для моделирования описанного процесса были заданы следующие параметры: напряжение

ISPC The Best of European Innovations, 
для «прямого» и «обратного» тока $4 \mathrm{~B}$, удельная проводимость электролита $0.21 / 0 \mathrm{O} \cdot \mathrm{cm}$, а также анодный и катодный выходы по току взяты равными 0.95 [9].

Согласно ограничениям на минимальную заданную толщину $\delta^{z a d}=10$ мкм (2) и максимальную продолжительность процесса $T^{\max }$ $=7200$ с (3), найдены матрицы активных анодных секций (4) и (5), обеспечивающие оптимальный режим функционирования анодных секций для «прямого» и «обратного» режимов нанесения гальванического покрытия соответственно:

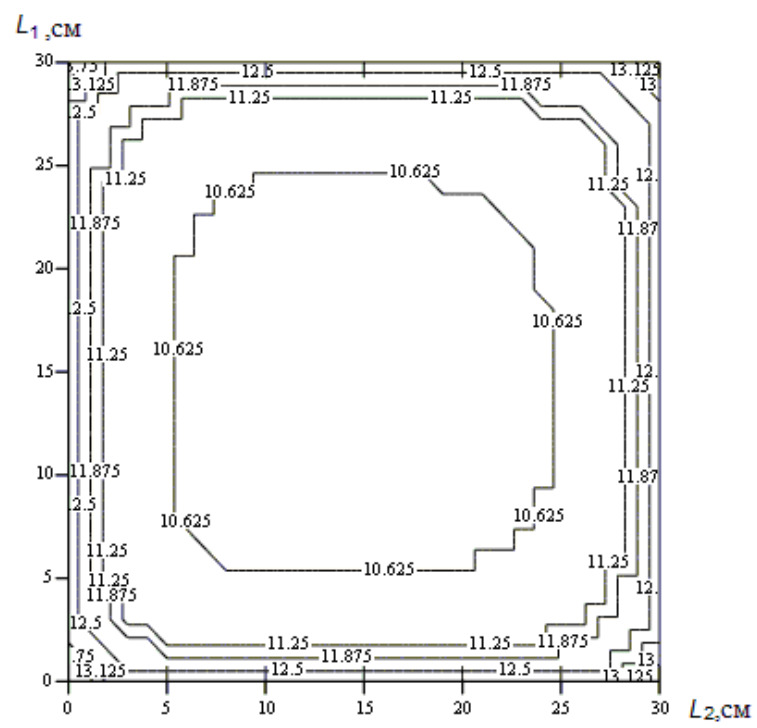

a)
$I_{1,2}^{\text {прям }}(\tau), I_{2,1}^{\text {прям }}(\tau), I_{2,2}^{\text {прям }}(\tau), I_{2,3}^{\text {прям }}(\tau), I_{3,2}^{\text {прям }}(\tau)$,
$I_{1,1}^{\text {обр }}(\tau), I_{1,3}^{\text {обр }}(\tau), I_{3,1}^{\text {обр }}(\tau), I_{3,3}^{\text {обр }}(\tau)$.
Остальные $\quad$ элементы находятся отключенном состоянии. При этом найденные оптимальные длительности включения реверсивного режима составляют $\tau^{\text {прям }}=7 \mathrm{c}$, $\tau^{\text {обр }}=2 \mathrm{c}$, а количество циклов переключения $v$ $=800$ раз. На рис. 1 представлено распределение толщины покрытия $\delta$ в мкм по поверхности изделия-катода для классического и предложенного режимов реверсирования.

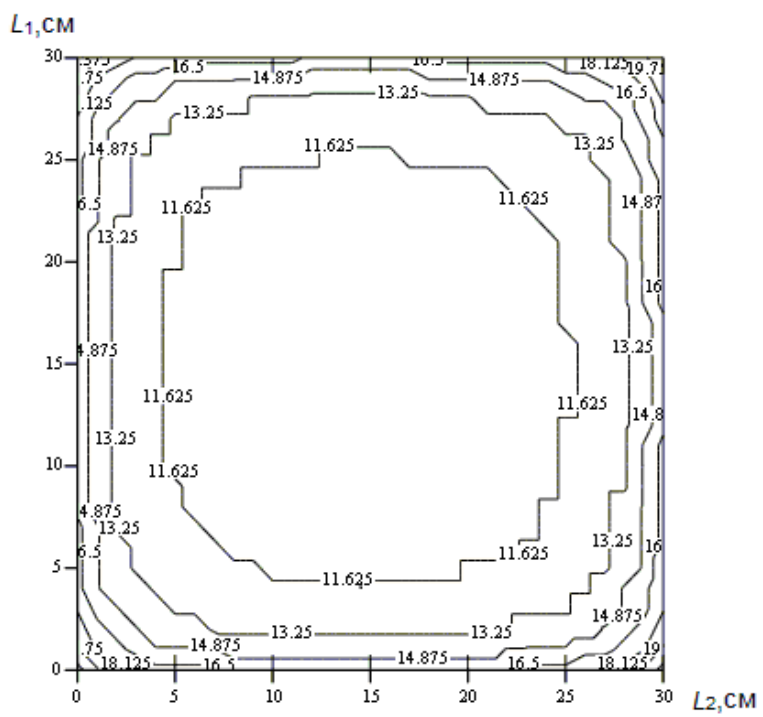

б)

Рисунок 1 - Распределение толщины покрытия $\delta$ в мкм по поверхности катода для различных режимов реверсирования: а) для предложенного режима; б) для классического режима.

Эффективность применения предлагаемого авторами способа модификации реверсивного режима может быть рассчитана по формуле:

$$
\ni=\frac{R_{1}-R_{2}}{R_{1}} \cdot 100 \%,
$$

где $R_{1}, R_{2}$ - критерии неравномерности распределения покрытия для классического и модифицированного режимов нанесения гальванического покрытия соответственно [10].

Согласно формуле (1) рассчитанные критерии неравномерности распределения толщины покрытия для классического режима реверсирования с монолитным анодом и его модификации составили соответственно $R_{1}=3.154$ и $R_{2}=2.428$. При этом вычисленная по формуле (6) эффективность предложенного способа по сравнению с классическим режимом реверса тока составила 23\%, что подтверждает применимость предлагаемой модификации существующего режима реверсирования тока для гальванической ванны со многими анодами. 


\section{References:}

1. Milovanov IV (2002) Modelirovanie i optimizatsiya tokovyih rezhimov $\mathrm{V}$ protsessah naneseniya galvanopokryitiy. Vestnik Tambovskogo gosudarstvennogo tehnicheskogo universiteta. 2002. - T.8, No 4. pp. 603-611.

2. Litovka YV, Romanenko AV, Afanasev AV (1998) Simulation and optimization of electroplating with current reversal. Theoretical Foundations of Chemical Engineering. 1998. vol. 32, no. 3. pp. 266 $-269$.

3. Konkina VV, Solovjev DS (2014) Matematicheskaya postanovka zadachi optimalnogo upravleniya galvanicheskim protsessom $\mathrm{v}$ reversivnom rezhime dlya mnogoanodnoy vannyi. Teoreticheskie i prakticheskie aspektyi tehnicheskih nauk: sb. statey mezhdunar. nauch.-prakt. konf. - Ufa: «Aeterna». 2014. pp. $41-43$.

4. Zenkevich O, Morgan K (1986) Konechnyie elementyi i approksimatsiya. - Moscow.: Mir, 1986. $-318 \mathrm{p}$

5. Bandi B (1988) Metodyi optimizatsii. Vvodnyiy kurs. - Moscow: Radio i svyaz, 1988. - 126 p.
6. Ekilik VV (2004) Elektrohimicheskie metodyi zaschityi metallov: metodicheskoe posobie po spetskursu. - Rostov-na-Donu, 2004. - 50 p.

7. Zemanová M, Krivosudská M, Chovancová M, Jorík $\mathrm{V}$ (2011) Pulse current electrodeposition and corrosion properties of Ni-W alloy coatings. Journal of Applied Electrochemistry. - September 2011. vol. 41. - pp. $1077-1085$.

8. Dini JW, Johnson HR (1980) The properties of gold deposits produced by DC, pulse and asymmetric AC plating. Gold Bulletin. - March 1980. - vol. 13. - pp. $31-34.1$.

9. Litovka YV, D'yakov IA (1997) Method to determine the potentials of anodes in a multianode electroplating bath. Theoretical Foundations of Chemical Engineering. 1997. Vol. 31, No. 2. pp. 191 -195 .

10. Solovjev DS, Litovka YV, Milovanov IV (2010) Sistema optimalnogo upravleniya galvanicheskoy vannoy $\mathrm{s}$ tsiklicheskim vklyucheniem anodnyih sektsiy. Radiotehnika. - 2010. - № 12. - pp. 44 - 48 . 